\title{
Anstrengungsinduziertes Asthma bei Fußballspielern im Alter von 8 bis 13 Jahren
}

\author{
M. Sidiropoulou \\ V. Tsimaras \\ E. Fotiadou \\ N. Aggelopoulou-Sakadami
}

\author{
Exercised-Induced Asthma in Soccer Players Ages from 8 to 13 Years
}

\section{Zusammenfassung}

Ziel der Studie war die Erforschung des anstrengungsinduzierten Asthmas (exercise-induced asthma, EIA) bei Fußball spielenden Kindern. Dreißig von hundert Jugendlichen der Fußballakademie von Panorama/Thessaloniki im Alter von 8 bis 13 Jahren wurden zufallsmäßig ausgesucht. Die Studie umfasste eine klinische Untersuchung, die Beantwortung eines Fragenbogens zur Gesundheitsanamnese der Kinder und den sechsminütigen Lauftest (free running test) mit Lungenfunktionsmessung durch Spirometrie. Vor und nach Beendigung des Testes wurde die Einsekundenkapazität ( $\mathrm{FEV}_{1}$, forced expiratory volume in one second) durch ein Mikro-Spirometer gemessen. Der Lauftest im Freien erfolgte mit einer Belastungsintensität im submaximalen Bereich (80-90\% max. heart rate). Die zurückgelegte Entfernung sowie die Werte der $\mathrm{FEV}_{1}$ in der Zeit 2', 5', 10', 15' und 30' nach dem Lauftest wurden registriert. Bei 12 (40\%) der 30 Kinder wurde ein $\mathrm{FEV}_{1}$-Abfall $>15 \%$ beobachtet. Dies war der Fall bei einem (11\%) von 9 Kindern, das keine Symptome in der Eigenanamnese, jedoch Asthma oder Allergie in der Familienanamnese aufwies, bei 3 von $12(25 \%)$ mit eigener Allergieanamnese und bei 8 von $9(89 \%)$ mit eigener Asthmaanamnese. Von den 12 Kindern mit EIA und einem $\mathrm{FEV}_{1}$-Abfall $>15 \%$ berichteten 9 über subjektive Symptome während des Lauftestes, aber nicht beim Fußballspielen. Der Lauftest im Freien erwies sich für die Diagnosestellung des EIA bei Fußballspielern im Kindesalter als erfolgreich und risikoarm. Ähnliche Studien sollten bei Spielern unterschiedlichen Alters und in unterschiedlichen Sportarten zur rechtzeitigen Entdeckung, Vorbeugung und Therapie des EIA durchgeführt werden.

\section{Abstract}

The purpose of this study was the detection of exercise induced asthma in soccer players aged 8-13 years. Thirty boys, 8-13 years old participated in the study. They were coming from an athletic team of north of Thessaloniki. The study included clinical examination, administration of a respiratory health questionnaire and the exercise - free running - test with spirometric measurements. Spirometric measurements were performed by using a microspirometer, before exercise and 2, 5, 10, 15 and $30 \mathrm{~min}$ after a 6 min free running exercise $(80-90 \%$ max heart rate). The highest forced expiratory volume in one second $\left(\mathrm{FEV}_{1}\right)$ value before exercise was compared with the lowest of post exercise values. The results showed a decline in $\mathrm{FEV}_{1}>15 \%$ in 12 out of 30 children. Particularly, decline in $\mathrm{FEV}_{1}$ was present in 1 (11\%) out of 9 children with free personal medical history but positive family history for asthma, in 3 (25\%) out of 12 children with allergies, and in $8(89 \%)$ out of 9 children with asthma. Symptoms were reported by 9 of 12 children with fall in $\mathrm{FEV}_{1}$ $>15 \%$, during the 6 min exercise test, who had no symptoms during the soccer games. Identification of EIA by exercise challenge test in young athletes is a useful component for the diagnosis of bronchial hyperresponsiveness. Similar studies should be performed on older and younger athletes who participate in different sports and games.

Institutsangaben

Aristoteles Universität Thessaloniki, Griechenland, Abteilung für Sportwissenschaft

und Körpererziehung, Labor für Physiologie und Entwicklungsmedizin

Korrespondenzadresse

Maria Sidiropoulou · Ionos Dragoumi 10 · 55236 Panorama · Thessaloniki · Griechenland ·

E-mail: sidirop@hotmail.com

Eingang: 11. August 2003 • Nach Revision alkzeptiert: 12. November 2004

Bibliografie

Pneumologie 2005; 59: 238-243 @ Georg Thieme Verlag KG Stuttgart · New York

DOI $10.1055 / \mathrm{s}-2004-830211$

ISSN 0934-8387 
Einleitung

Anstrengungsinduziertes Asthma (exercise-induced asthma, EIA) ist definiert als akute Verengung der Atemwege während oder unmittelbar nach starker körperlicher Belastung auf der Grundlage einer bronchialen Hyperreaktivität, es imponiert als vollständig reversible bronchiale Obstruktion, und stellt eine klinische Form des Asthma bronchiale dar [12].

Ätiologie und Pathogenese des EIA sind bis heute unklar geblieben bzw. nicht gänzlich geklärt. Übereinstimmung besteht in der Auffassung, dass die bronchiale Obstruktion in enger Beziehung zu den Temperaturverhältnissen in den intrathorakalen Luftwegen steht. Anstrengungsasthma entsteht vor allem in kalter, trockener Luft, wenn den Nasen und der Bronchialschleimhaut Wärme und Feuchtigkeit entzogen werden, was eine Hyperämie bewirkt. Wärme und Wasserverlust sind die Folgen der belastungsbedingten Hyperventilation. Eine weitere mögliche Ursache ist die Osmolaritätsänderung im Schleimfilm, der das Bronchialepithel bedeckt. Ein hoher Adrenalinspiegel kann die Obstruktion beeinflussen, für eine „Mediatorenhypothese“ spricht die auch lange nach Beendigung der körperlichen Belastung andauernde Atemnot sowie das Phänomen einer Refraktärperiode bei Mehrfachbelastung. Kurz aufeinander folgende intervallmäßige Anstrengungen (Warm up) verursachen eine geringere bronchiale Reaktion, während virale Atemwegsinfekte das EIA verstärken $[13,18,20,24,25]$. Die wesentlichen pathophysiologischen Mechanismen, die zum anstrengungsinduzierten Asthma beitragen und den Grad der Bronchialreaktion bestimmen, sind die Luftfeuchtigkeit und die Temperatur der eingeatmeten Luft sowie die Belastungsart, -intensität, -dauer und -methode während der sportlichen Anstrengung [3,6,12,19,21].

Zwar kann bereits bei gesunden Kindern und Erwachsenen, bei den Verwandten von Asthmatikern und bei Patienten mit Heuschnupfen eine gewisse anstrengungsinduzierte Labilität der Atemwege beobachtet werden, doch lässt sich das EIA vor allem bei Patienten mit bronchialer Hyperreagibilität nachweisen $[12,24]$.

Generell stellen Mannschaftsspiele nicht kalkulierbare und daher auch nicht dosierbare Belastungsformen dar. Mannschaftsspiele bedürfen wegen ihres Wettkampfcharakters besonderer Fähigkeiten der Koordination und der Kondition, was zur Überanstrengung und eventuell zur Auslösung eines Asthmaanfalles bei Personen mit Hyperreagibilität der Bronchien führen kann $[8,12]$. So ist es gerade auch das Fußballspielen, eine Mannschaftssportart mit hoher Laufbelastung, bei der solche Überanstrengungen häufig auftreten. Während des Spiels werden die Kinder vom schnellen Wechsel zwischen Abwehr und Angriff sowie vom intensiven Zusammenspiel der Spieler sehr beansprucht [26]. Diese hohe Belastungsintensität stellt ein großes Handikap für den Spieler mit bronchialer Hyperreagibilität dar und die Gefahr der Manifestation des Bronchospasmus ist erhöht $[7,12]$. Forschungen haben gezeigt, dass während des Spieles die Kraftentwicklung und die Laufleistung groß sind. Die Kinder zwischen 8-12 Jahren legen im Durchschnitt bei einem Wettkampf von 50 min ( $2 \times 25$ min) Dauer 4230 m zurück. Während des Spiels sind Laktatanstiege auf bis zu $8,3 \mathrm{mmol} / \mathrm{l}$ gemessen worden, was die hohe Belastungsintensität bestätigt $[1,26]$.
In Fällen eines nicht diagnostizierten EIA's glauben viele Kinder, dass ihre Symptome, wie Dyspnoe, Brustschmerz, Kurzatmigkeit usw. auf schlechte Kondition zurückzuführen sind, sodass die Erkrankung nicht erkannt wird [10,20].

Erfahrungen mit Sportlern weisen darauf hin, dass EIA erst bei sehr hoher Belastungsintensität auftritt und gelegentlich der Aufdeckung unter Laborbedingungen immer dann entgeht, wenn der respiratorische Wärmeaustausch nicht die in praxi auftretenden Werte erreicht. Bei diesen Patienten sollte das EIA in Form des situativen Exercise-Tests unter den Bedingungen provoziert werden, unter denen es sich natürlicherweise manifestiert. Mit diesem Verfahren ist oft auch bei einem negativen Laufbandergebnis ein Anstrengungsasthma nachweisbar. Dabei haben sich tragbare Lungenfunktionsmessgeräte, die die Bestimmung des exspiratorischen Spitzenflusses (PEFR) oder der Einsekundenkapazität $\left(\mathrm{FEV}_{1}\right)$ gestatten, bewährt. Damit ist es auf einfache Weise möglich ein EIA festzustellen, therapeutische Maßnahmen individuell anzupassen, ihre Wirksamkeit unter Trainings- und Wettkampfbedingungen zu überprüfen und die Einflüsse von saisonalen Inhalationsallergenen sowie von physikochemischen Umweltreizen zu bestimmen [2, 5, 17, 24,28].

Ziele der vorliegenden Arbeit waren es, die Häufigkeit des Auftretens eines EIA bei Fußballspielern im Alter zwischen 8-13 Jahren aufzudecken sowie Faktoren zu bestimmen, die das Auftreten der Krankheit beeinflussen.

Die Untersuchungen wurden im Labor für Physiologie und Entwicklungsmedizin der Abteilung für Sportwissenschaft und Körpererziehung der Aristoteles Universität von Thessaloniki und auf dem Fußballplatz des Vereins durchgeführt.

\section{Material und Methoden}

An der Studie nahmen 30 Kinder im Alter zwischen 8-13 Jahren teil, die der Fußballakademie von Panorama angehören und mit ihren Familien im Zentrum oder im Osten von Thessaloniki leben. Sie wurden unter 100 Kindern, die in der gleichen Akademie trainierten, ausgewählt (einfache Zufallsstichproben - simple random sampling). Die Studie beinhaltete eine klinische Untersuchung der Kinder, das Ausfüllen eines Fragenbogens (siehe Anhang) und den Lauftest im Freien. Die Eltern und Kinder wurden über Planung und Ziel der Studie, sowie Durchführung der Untersuchungen und Messungen aufgeklärt.

\section{Klinische Untersuchung}

Sie umfasste die Anamneseerhebung (Familien und Eigenanamnese), die Ermittlung von Körpergewicht und Körpergröße, das Ausfüllen eines Fragebogens durch Eltern und Kinder, die klinische Untersuchung einschließlich eines EKG und einer Ultraschalluntersuchung des Herzens. Kinder mit Herzerkrankungen oder Übergewicht wurden von der Studie ausgeschlossen. Von den Eltern, die auch während der klinischen Untersuchung anwesend waren, stammten die Informationen zur Anamnese. 
Fragebogen

1. Zeigt das Kind allergische Reaktionen?

2. Sind Sie Allergiker?

3. Welches sind die Allergene des Kindes oder ihre eigenen?

4. Nehmen Sie Medikamente?

5. Hatte das Kind während der letzten Tage erhöhte Temperaturen?

6. Hatte das Kind innerhalb der letzten zwei Wochen Erkältung mit

akuter Rinitis?

7. Hatte das Kind in der Vergangenheit Bronchialasthma?

8. Entwickelte das Kind Dyspnoe, Keuchen/Pfeifen/Husten, Brustschmerz

oder andere Atemprobleme?

9. Hatte das Kind früher Pneumonie bzw. andere Lungenerkrankungen?

10. Früher Krankenhausaufenthalte? Durch welche Krankheiten bedingt?

11. Musste das Kind das Laufen abbrechen? Nach welcher Distanz?

12. Entwickelte das Kind Atemsymptome nach Dauerlauf und war

Erholung nötig?

13. Hat jemand in der Familie Bronchialasthma oder Allergien?

14. Raucht jemand in der Familie?

15. Wie viele Tage fehlte das Kind während des letzten Schuljahres

wegen Atemproblemen?

Abb. 1 Fragebogen für die Eltern.

\section{Der Fragebogen}

Den Eltern wurde ein Fragebogen zur Gesundheitsanamnese der Kinder, insbesondere zur Allergie- bzw. Asthmaanamnese und zu anderen Lungenproblemen vorgelegt (siehe Abb.1). Die Fragen, die Zusammenhänge zwischen Ermüdung und anderen Symptomen während der Dauer des Fußballspieles betrafen, wurden von den Kindern selbst und den Trainern beantwortet. Der Grad der Umweltverschmutzung wurde nicht erfasst; die Kinder hielten sich jeden Tag im Großraum Thessaloniki auf und lebten unter vergleichbaren Umweltbedingungen. Die Raucheranamnese der Familien wurde registriert.

\section{Durchführung des Belastungstests zur Feststellung des EIA's durch Laufen im Freien und Spirometrie Messungen}

Der Lauftest im Freien beinhaltete eine Anfangsspirometrie (Ruhewertmessung) mit Bestimmung des $\mathrm{FEV}_{1}$ durch das MikroSpirometer der Firma Micro Medical. Das Mikro-Spirometer ist klein, leicht und mobil und für die Asthmadiagnose geeignet, bietet ein hohes Niveau an Genauigkeit $( \pm 3 \%)$ und Reproduzierbarkeit bei einfacher Bedienung. Es verfügt über einfache Kalibrierungsüberprüfung und über einen digitalen Mikro-MedicalVolumenwandler, der Genauigkeit, Stabilität und Zuverlässigkeit gewährleistet.

Eine Woche vor dem Lauftest wurde die Funktion des Mikro-Spirometers demonstriert und den Testpersonen die richtige Technik beim Ein- bzw. Ausatmen beigebracht $[2,9,19]$. Jede Testper- son führte drei Versuche aus und der beste wurde registriert. Von den Kindern wurde verlangt, am Tag der Durchführung des Lauftests nicht am Sportunterricht in der Schule teilzunehmen, sodass sie sich während des Tests nicht in der Refraktärperiode befanden $[11,22]$.

Das Laufen wurde „kalt“ gestartet. Durch eine möglichst früh erzielte hohe Belastungsintensität sollte die Auslösungswahrscheinlichkeit eines Anstrengungsasthmas erhöht werden. Die rasch erreichte angestrebte Belastung wurde 6 Minuten lang aufrechterhalten. Vor Beginn des Laufens wurden keine Aufwärmübungen durchgeführt, weil gezeigt werden konnte, dass man mit intervallartigen Aufwärmübungen dem Anstrengungsasthma vorbeugen kann $[13,18]$.

Keines der Kinder nahm regelmäßig Medikamente ein. Eine Woche vor dem Test wurden von den Kindern auch bedarfsweise keine Asthma-Medikamente mehr eingenommen, damit das Testergebnis nicht beeinflusst wurde.

Die vor dem Start des Lauftests registrierten $\mathrm{FEV}_{1}$-Ruhewerte zeigten bei keinem der Untersuchten pathologische Werte. Es folgte ein 6-minütiger Lauftest im Freien bei einer Belastungsintensität von 80 - 90\% der maximalen Herzfrequenz. Um den konstanten Laufrhythmus bei einer Herzfrequenz von 170-180 Schlägen pro Minute zu stabilisieren, wurden Herzfrequenzmesser (Polar Vantage, NV HRM der Firma Polar Electro Oy, Finnland) benutzt. Um eine konstante Belastungsintensität im submaximalen Bereich zu gewährleisten, regulierte der erfahrene Trainer im Folgenden außerdem die Laufgeschwindigkeit, indem er mit der Versuchsperson lief und den Testablauf erleichterte. Die zurückgelegte Strecke wurde registriert. Alle Symptome, wie subjektive Atemnot oder Brustschmerz, objektivierbare Symptome wie z.B. Husten, sowie die Unterbrechung des Testes usw. wurden registriert. Lungenfunktionsmessungen erfolgten vor Belastung (Ruhespirometrie) sowie 2, 5, 10, 15 und 30 Min. nach Belastungsende $[4,14,16,23]$. Als Leitparameter diente die Veränderung der Einsekundenkapazität $\left(\mathrm{FEV}_{1}\right)$ gegenüber dem Ausgangswert. Dazu wurde die Differenz des niedrigsten $\mathrm{FEV}_{1}$ (nach Belastung) zum Ausgangswert in Prozent nach folgender Formel berechnet:

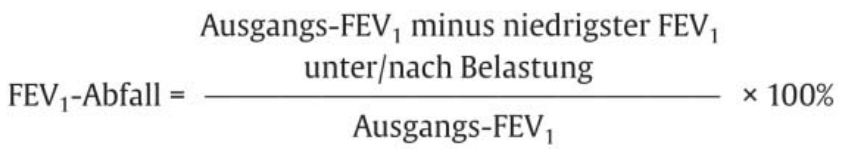

Die Messungen fanden zur gleichen Uhrzeit und unter gleichen klimatischen Bedingungen im Mai statt. Die Temperatur betrug $21^{\circ} \mathrm{C}$ bis $25^{\circ} \mathrm{C}$ und die Luftfeuchtigkeit lag zwischen $40 \%-45 \%$.

Für die Analyse und Auswertung der Daten wurde die deskriptive Statistik angewendet. Für den Vergleich von Anamnese und Schweregrad des $\mathrm{FEV}_{1}$-Abfalls beim EIA wurde die statistische Analyse $\chi^{2}$ benutzt. In dieser Studie wurde als Anstrengungsasthma und damit als pathologischer Befund definiert, wenn die $\mathrm{FEV}_{1}$ um mehr als $15 \%$ gegenüber dem Ausgangswert abfiel $[4,12]$. Die Normalwerte der $\mathrm{FEV}_{1}$ für Kinder wurden anhand der Tabelle des Lungenfunktionsrechners des Mikro-Spirometers berechnet. 


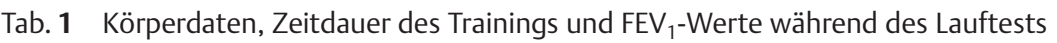

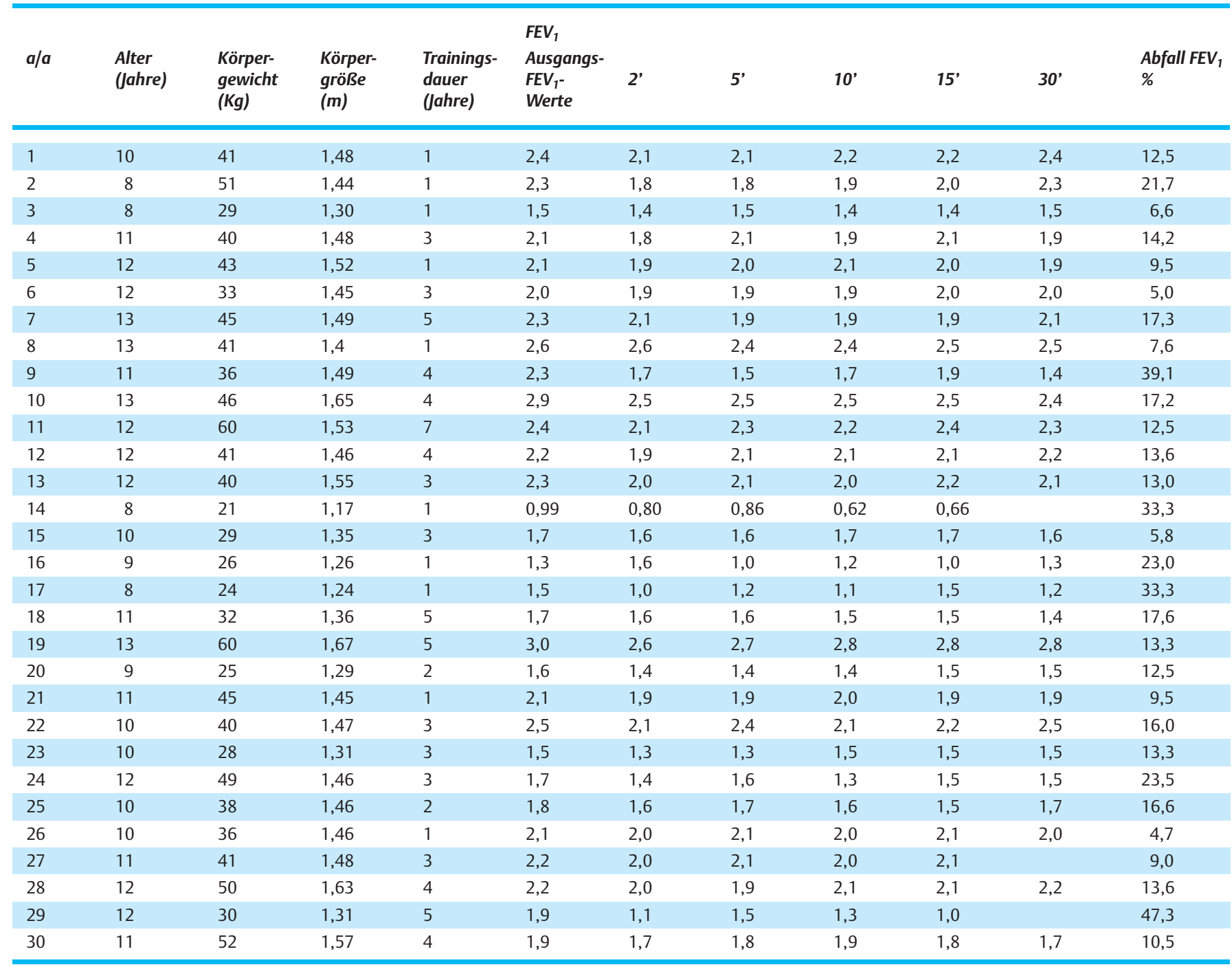

\section{Ergebnisse}

Die körperlichen Daten der Untersuchten wie Gewicht und Größe sowie die Messungen der $\mathrm{FEV}_{1}$ (Ruhe und Testwerte) wurden registriert und in der Tab. 1 zusammengefasst. Bei keinem der Kinder wurden Abweichungen der $\mathrm{FEV}_{1}$-Ruhewerte von den Normalwerten festgestellt. Ein Vergleich der klinischen Anamnese und des $\mathrm{FEV}_{1}$-Abfalls ergab, dass insgesamt zwölf Kinder einen $\mathrm{FEV}_{1}$-Abfall $>15 \%$ (40\%) und 18 Kinder einen $\mathrm{FEV}_{1}$-Abfall $<15 \%$ (60\%) aufwiesen (Tab. 2). Drei Kinder wiesen einen FEV F $_{1}$-Abfall schon während der 2-min-Messung, ein Kind während der 5-min-, zwei während der 10-min-, drei während der 15minund drei während der 30-min-Messung auf. Während des Lauftests und nach dessen Beendigung entwickelten 15 Kinder Asthmasymptome (Hustenanfall, Dyspnoeempfinden, Brustschmerz). Von den 15 Kindern mit Asthmasymptomen zeigten 9 einen $\mathrm{FEV}_{1}$-Abfall $>15 \%$, während 6 Kinder mit Symptomen einen $\mathrm{FEV}_{1}$-Abfall $<15 \%$ aufwiesen. Drei der 12 Kinder mit einem $\mathrm{FEV}_{1}$-Abfall > $15 \%$ wiesen keine Symptome auf. Von den drei Kindern ohne Symptome und mit einem $\mathrm{FEV}_{1}$-Abfall > $15 \%$ hatte ein Kind eine symptomfreie Eigenanamnese. Es wurde kein Zusam- menhang zwischen der Zahl der Trainingsjahre und den $\mathrm{FEV}_{1}$-Werten festgestellt.

Tab. 2 zeigt die Beziehungen zwischen anamnestischen Angaben zum Vorliegen von Allergien und eines Asthma bronchiale sowie dem $\mathrm{FEV}_{1}$-Abfall unter Belastung. Von den dreißig Kindern waren $9(30 \%)$ an Asthma erkrankt, hatten rezidivierende Anfälle oder hatten in den letzten Jahren eine eigene Asthmaanamnese. Acht $(89 \%)$ von ihnen zeigten beim Lauftest Symptome (Testantwort $\mathrm{FEV}_{1}$-Abfall > 15\%). Zwölf Kinder hatten eine Pollen-, Medikamenten-, Hausstaub- oder Tierhaarallergie. Von diesen 12 Kindern (40\%), zeigten drei einen $\mathrm{FEV}_{1}$-Abfall > $15 \%$ (25\%). Neun von den 30 untersuchten Kindern hatten eine symptomfreie Eigenanamnese, 6 von ihnen hatten eine positive Familienanamnese, davon 3 mit Asthma- und 3 mit Allergiesymptomen. Eines der 3 Kinder mit Asthmasymptomen in der Familienanamnese wies einen $\mathrm{FEV}_{1}$-Abfall $>15 \%$ auf. Von den 9 Untersuchten mit symptomfreier Eigenanamnese wies nur ein Kind (11\%) einen $\mathrm{FEV}_{1}$-Abfall $>15 \%$ auf. Die statistische Analyse $\chi^{2}$ zeigte, dass eine positive Asthmaanamnese mit der Stärke des FEV $_{1}$-Abfalls $\left(\chi^{2}=17.93, \mathrm{P}=.006\right)$ korrelierte. 
Tab. 2 Beziehungen zwischen anamnestischen Angaben zum Vorliegen von Allergien und eines Asthmas bronchiale sowie dem FEV ${ }_{1}$-Abfall unter Belastung

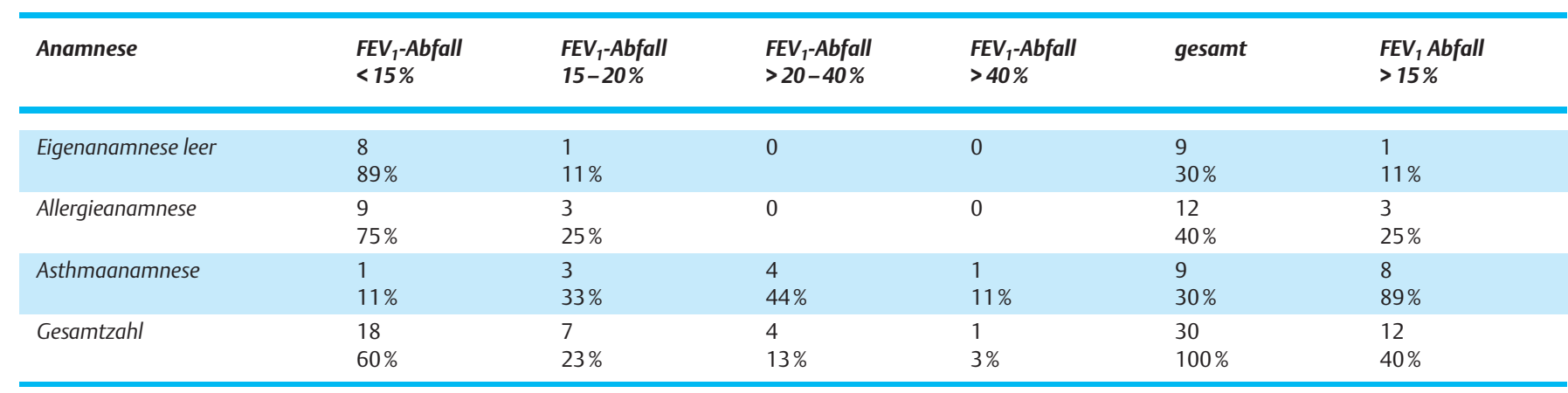

Neun von 17 Kindern rauchender Eltern zeigten eine bronchiale Hyperreagibilität und hatten bei dem Belastungstest einen Abfall $>15 \%$, gegenüber nur 3 von 13 Kindern nicht rauchender Eltern.

\section{Diskussion}

Diese Studie zeigte, dass von den dreißig Kindern 12 (40\%) einen $\mathrm{FEV}_{1}$-Abfall $>15 \%$ des Ausgangswertes und somit eine bronchiale Hyperreaktivität während der Testbelastung aufwiesen. Ein $\mathrm{FEV}_{1}$-Abfall $>15 \%$ zeigte sich bei einem ( $\left.11 \%\right)$ von 9 Kindern mit symptomfreier Eigenanamnese, bei $3(25 \%)$ von 12 Kindern mit Allergieanamnese und bei 8 (89\%) von 9 Kindern mit Asthmaanamnese. Sieben Kinder litten an leichtem EIA mit einem $\mathrm{FEV}_{1}$-Abfall von $15-20 \%$, vier an mittelschwerem $(20-40 \%)$ und eins an schwerem EIA mit einem $\mathrm{FEV}_{1}$-Abfall von über $40 \%$.

Die Entwicklung allergischer Reaktionen hängt mit der Exposition der Kinder gegenüber verschiedenen Allergenen zusammen. $[16,17]$. Ein Viertel der Allergiker entwickelt auch Bronchialasthma. Gustovic u. Mitarb. [6] fanden in ihrer Studie, dass dreißig bis vierzig Prozent der atopischen Kinder ohne klinisches Asthma bei körperlicher Belastung mit Symptomen reagierten. Der $\mathrm{FEV}_{1}$-Abfall war wesentlich größer als bei der Gruppe, die keine Allergie aufwies. Eliasson u. Mitarb. [3] stellten fest, dass neun von zwanzig Untersuchten mit Allergiesymptomen (Nahrungsmittel-, Medikamenten-, saisonale Allergie) einen anstrengungsinduzierten Bronchospasmus entwickelten, wenn sie durch die Technik der Hypokapnie - Hyperventilation (EVH - eucapnic voluntary hyperventilation) mit kalter und trockener Luft untersucht wurden. Einen Abfall der $\mathrm{FEV}_{1}>15 \%$ zeigten in dieser Studie drei von zwölf Kindern (25\%) mit Allergien. Kukafka u. Mitarb. [11] und Randolf \& Faser [20] fanden einen sehr engen Zusammenhang zwischen einer Eigenanamnese mit Asthma und EIA bei Sportlern an Gymnasien.

Die Häufigkeit des EIA bei Kindern mit bereits diagnostiziertem Asthma wird in anderen Publikationen mit 70-90\% angegeben [9,17]. Bei Personen ohne Asthmaanamnese können ebenfalls Asthmasymptome nach Belastung auftreten, die Häufigkeit erreicht bei Sportlern 9\% [14].

Der Belastungstest durch freies Laufen als natürliches Provokationsmittel stellt die meistakzeptierte Methode und eine verbrei- tete Technik zum Nachweis der bronchialen Hyperreagibilität in der Diagnostik des EIA's dar [3,11,15,23,27]. Auch der 6-minütige Lauftest nach Rasmussen u. Mitarb. [21] ist in der Diagnostik des EIA's sowohl bei Kindern mit erhöhtem Risiko als auch bei Kindern, die keine Symptome aufweisen, sehr zuverlässig. Für den Fall, dass sich durch den Belastungstest keine Symptome reproduzieren lassen, muss ein inhalativer Provokationstest mit pharmakodynamischen Substanzen wie Histamin, Metacholin oder Carbachol durchgeführt werden, damit ein mögliches EIA nicht unerkannt bleibt [24].

In unserer Untersuchung zeigte eines von neun (11\%) Kindern aus symptomfreien Familien und ohne Eigenanamnese einen $\mathrm{FEV}_{1}$-Abfall $>15 \%$. Tsanakas u. Mitarb. [27] erforschten das EIA bei Kindern im Schulalter, aber nicht bei Sportlern, mit der gleichen Methode. Feinstein u. Mitarb. [4] untersuchten Fußballspieler mittels Spirometrie und Steptest und fanden bei $44 \%$ einen Abfall der Lungenfunktionsgrößen PEFR (Peak expiratory flow rate) und $\mathrm{FEV}_{1}$.

Weiler u. Mitarb. [28] entdeckten, dass Fußballspieler der höheren Liga in Kalifornien, die unter Asthmasymptomen litten, ohne ihnen Bedeutung beizumessen, während der Vorbereitung auf die Spiele ein EIA entwickelten. Rupp u. Mitarb. [22] kamen zum Ergebnis, dass 29\% der Sportler von Gymnasien und Lyzeen während des Lauftestes einen Bronchospasmus entwickelten, ohne es zu wissen. Alle o.g. Sportler hatten ein nicht diagnostiziertes EIA.

Unsere Studie zeigte, dass 9 von 15 Kindern, die Hustensymptome, Atemnot, Erschöpfung oder Brustschmerz hatten, einen $\mathrm{FEV}_{1}$-Abfall > 15\% aufwiesen. Die 6 Kinder, die Symptome entwickelten, aber keinen $\mathrm{FEV}_{1}$-Abfall aufwiesen, müssen weiteren Untersuchungen unterzogen werden. Bemerkenswert ist, dass die Kinder die Symptome beim Fußballspielen nicht als Krankheitssymptome werteten. Das Anstrengungsasthma ist in seiner Frühform oft nur als sportlicher Leistungsknick ohne wesentliche Dyspnoe erkennbar [24].

In unserer Studie zeigte sich, dass neun von 17 Kindern rauchender Eltern gegenüber 3 von 13 Kindern nicht rauchender Eltern EIA entwickelten. Studien haben gezeigt, dass aktives und passives Rauchen die Entwicklung von Allergie, bronchialer Hyperre- 
aktivität und sogar Asthma, begünstigt. Kinder, deren Eltern rauchen, sind dabei besonders gefährdet [22].

Mehrere Studien haben bewiesen, dass die Diagnostik mittels Lauftest im Freien temperaturabhängig ist. Bei gleicher Testanordnung im Winter wäre wahrscheinlich ein höherer Anteil von Kindern gefunden worden, die auf den Belastungstest mit Symptomen reagiert hätten [12]. Provoziert man Patienten mit EIA lediglich durch Inhalation kalter Luft, so lässt sich zeigen, dass die bronchiale Obstruktion umso stärker ausfällt, je trockener die Einatmungsluft ist [24]. Der Einfluss der Position des Fußballspielers auf dem Platz auf die bronchiale Hyperreagibilität oder auf die auftretenden Symptome müsste noch eruiert werden.

Was die Ergebnisse unserer Studie betrifft, so lässt sich sagen, dass Kinder mit EIA, die aktiv Fußball trainierten, zufriedenstellende Leistungen brachten, was sich wahrscheinlich darauf zurückführen lässt, dass die Spielanforderungen in dieser Alterstufe entweder nicht hoch waren, oder aber nur von kurzer Dauer, so dass die Belastungsgrenze nicht erreicht wurde. Auch die häufigen Auswechselungsmöglichkeiten während des Spiels sowie die kleinere Größe der Fußballfelder mögen dazu beigetragen haben. Anderseits aber wurden auftretende Symptome entweder nicht wahrgenommen oder falsch gedeutet.

Zusammenfassend wäre zu betonen, dass Kinder mit EIA körperliche Belastung nicht meiden sollten, da mit adäquater Therapie, Prophylaxe und angepasster Belastung die Symptome des EIA beherrscht werden können. Dadurch wird langfristig die Kondition verbessert und die Dauer der sportlichen Betätigung gesteigert. In dieser Studie erwies sich das Laufen im Freien für die Diagnosestellung des EIA bei Fußball spielenden Kindern als erfolgreich und risikoarm.

\section{Literatur}

${ }^{1}$ Bangsbo J. Fitness training in Football. A scientific approach. Bagsvaerd: $\mathrm{HO}+$ Strom, 1994

${ }^{2}$ Einright T. Exercise-induced asthma and the asthmatic athlete. Wiscon Med J 1996; 95: 375 - 378

${ }^{3}$ Eliasson M, Phillips D, Krishanan R. Sensitivity and Specificity of Bronchial provocation testing. Chest 1992; 112: $347-355$

${ }^{4}$ Feinstein RA, Russa J, Wand-Dohlman A. Screening adolescent athletes for exercise induced asthma. J Clin Sports Med 1996; 6: 119-123

${ }^{5}$ Fjellhirkeland L, Galsvik A, Wolloe A. Swimming induced asthma. Tridsskrift for Den Morske Laegeforening 1995; 115: 2051 - 2053
${ }^{6}$ Gustovic A, Arifhodzic N, Robinson A et al. Exercise Testing Revisited. Chest 1994; 7105: 1127-1131

${ }^{7}$ Haber P. Bewegungstraining in der Rehabilitation von Patienten mit chronischer Atemwegserkrankung. Prax Klin Pneumol 1988; 42: $625-627$

${ }^{8}$ Jones A, Brawn M. Severing for childhood asthma using an exercise test. British J Gen Pract 1999; 44: 127-131

${ }^{9}$ Kattan M, Keens TC, Mellis CM et al. The response to exercise in normal and asthmatic children. J Pediatr 1978; 92: 718 - 721

${ }^{10}$ Kilvington H. Exercise and Asthma. Feature 1998; 8: 37 - 39

${ }^{11}$ Kukafka D, Lang D, Porter S et al. Exercise induced Bronchospasm in High School Athletes via a Free Running Test. Chest 1998; 114: $1613-1622$

${ }^{12}$ Lecheler J, Biberger A, Pfannbecker BB. Asthma \& Sport. Theoretische Grundlagen und praktische Handlungsanleitungen. Tübingen: Ina Verlag, 1997

${ }^{13}$ Lecheler J, Biberger A, Seligmann C et al. Sporttherapie in der Behandlung des kindlichen Asthma bronchiale. Vergleich von Intervall- und Dauerlauftraining. Prax Klin Pneumol 1988; 42: 475-478

${ }^{14}$ Milgrom H, Taussig LM. Keeping children with exercise induced asthma active. Pediatrics 1999; 3: $104-110$

${ }^{15}$ Neder A, Nery E, Silva A et al. Short term effects of aerobic training in the clinical management of moderate to severe asthma in children. Thorax 1999; 54: $202-206$

${ }^{16}$ Ng'ang'a W, Odhiambo JA, Mungai $M$ et al. Prevalence of exercise induced bronchospasm in Kenyan school children: An urban rural comparison. Thorax 1998; 53: 919-926

${ }^{17}$ Ninan TK, Rusell G. Is exercise testing useful in community based asthma survey? Thorax 1993; 48: 1218-1221

${ }^{18}$ Pfannebecker B. Vergleichende Untersuchung verschiedener Aufwärmmethoden in ihrer Auswirkung auf das Anstrengungsasthma bei Kindern und Jugendlichen. München, 1993

${ }^{19}$ Randolf C. Exercise-induced asthma: Update on pathophysiology, clinical diagnosis and treatment. Cur Probl Pediatr 1997; 27: 53 - 77

${ }^{20}$ Randolph C, Fraser B. Ongoing Evaluation of Questionnaire (Q) for exercise Asthma. Ann Allergy 1992; 67: 117-121

${ }^{21}$ Rasmussen F, Lambrechtsen J, Siersted HC et al. Asymptomatic bronchial hyperresponsiveness to exercise in childhood and the development of asthma related symptoms in young adulthood: The Odense Schoolchild Study. Thorax 1992; 54: 587 - 589

${ }^{22}$ Rupp MT, Brudno SB, Gull MF. The value of screening for risk of exercise induced asthma in high school athletes. Ann Allergy 1993; 70 : $339-342$

${ }^{23}$ Schied S, Wang-Dohman A. Incidence of exercise-induced Bronchospasm in high school football players. N Engl J Med 1991; 19: $1362-1367$

${ }^{24}$ Schmitz-Schumann M, Matthys H, Menz G et al. Anstrengungsinduziertes Asthma. Dt Zeitschr Sportmed Sonderheft 1985; 6: $42-47$

25 Spector SL. Update on exercise-induced asthma. Ann Allergy 1993; 6 : $571-577$

${ }^{26}$ Tritschocks J. Untersuchung über die Laufbelastung von 6 - 10jährigen jugendlichen Fußballspielern. Fußballtraining 1996; 2: 3-10

27 Tsanakas J, Milner N, Banister RDC et al. Free running asthma screening test. Arch Dis Child 1988; 63: 261 - 265

${ }^{28}$ Weiler M, Metzger W, Arlene L et al. Prevalence of Bronchial Hyperresponsiveness in Highly Trained Athletes. Chest 1986; 90: 23 -29 